\title{
Damping scales of neutralino cold dark matter
}

\author{
Stefan Hofmann ${ }^{a *}$, Dominik J. Schwarz ${ }^{a, b \dagger}$, Horst Stöcker ${ }^{a}$ \\ ${ }^{a}$ Institut für Theoretische Physik, J.W.Goethe-Universität, Robert-Mayer-Str. 8-10, D-60054 Frankfurt a.M., Germany \\ ${ }^{b}$ Institut für Theoretische Physik, Technische Universität Wien, Wiedner Hauptstraße 8-10, A-1040 Wien, Austria
}

(October 31, 2018)

\begin{abstract}
The lightest supersymmetric particle, most likely the neutralino, might account for a large fraction of dark matter in the Universe. We show that the primordial spectrum of density fluctuations in neutralino cold dark matter (CDM) has a sharp cut-off due to two damping mechanisms: collisional damping during the kinetic decoupling of the neutralinos at about $30 \mathrm{MeV}$ (for typical neutralino and sfermion masses) and free streaming after last scattering of neutralinos. The last scattering temperature is lower than the kinetic decoupling temperature by one order of magnitude. The cutoff in the primordial spectrum defines a minimal mass for CDM objects in hierarchical structure formation. For typical neutralino and sfermion masses the first gravitationally bound neutralino clouds have to have masses above $10^{-7} M_{\odot}$.

PACS numbers: 14.80.Ly, 98.35.Ce, 98.80.-k, 98.80.Cq
\end{abstract}

\section{INTRODUCTION}

Recent observations of the cosmic microwave background (CMB) anisotropies are consistent with a key prediction of inflationary cosmology: The universe appears to be spatially flat [1]. However, only a small percentage of the mass that is needed to account for the critical energy density of the universe comes in the form of baryons. Recent observations of primordial deuterium and other light elements suggest that the baryonic mass density is $\omega_{\mathrm{b}}=0.019 \pm 0.0024$ [2], which implies that only about 5 per cent of the mass in the universe is baryonic. The remaining mass is assumed to be a mixture of different forms of yet unknown dark matter and dark energy. However, we do have evidence, mainly from the study of large scale structures, about the properties of dark matter.

Cold dark matter (CDM) by definition has a nonrelativistic equation of state at the beginning of structure formation around the matter-radiation equality [3]. For successful structure formation an important fraction of the dark mass has to be cold dark matter. Although purely baryonic matter and hot dark matter (relativistic equation of state at matter-radiation equality) models have been ruled out long ago [4], a model with a cosmological constant and baryonic matter only provides a good fit to the recent CMB observations [5]. When combined with other cosmological observations it turns out that the small sound speed (at photon decoupling) of the baryonic matter can only be compatible with the observed multipole moments if the universe is closed [6]. Moreover, this model does not provide enough power at

\footnotetext{
*stehof@th.physik. uni-frankfurt.de

${ }^{\dagger}$ dschwarz@hep.itp.tuwien.ac.at
}

small scales to explain the observed distribution of galaxies $[6]$.

The most important feature of CDM is hierarchical structure formation, i. e., small structures form first and grow to larger structures later. A natural question obviously is: What is the smallest mass scale [7] of CDM structures?

Since the nature of CDM is unknown, the answer to this question will not be the same for different CDM candidates. Natural candidates are particles that are predicted by extensions of the standard model of particle physics. A CDM candidate should be (meta-)stable, (electrical and color) neutral, and heavy.The minimal supersymmetric standard model (MSSM) with the assumption of conserved R-parity provides an excellent candidate: the lightest neutralino $\tilde{\chi}_{1}^{0}$, which probably is the lightest supersymmetric particle (LSP) [8].

The time of kinetic decoupling of CDM depends on the nature of CDM 9 11]. During kinetic decoupling collisional damping is the dominant mechanism. Once CDM is fully decoupled from the radiation fluid, damping due to free streaming happens. Interesting general considerations on damping mechanisms for CDM have been published by Boehm, Fayet, and Schaeffer [11] recently.

After the neutralinos decouple chemically (at about $\left.T_{\text {cd }} \sim M_{\tilde{\chi}} / 20\right)$ they remain in kinetic equilibrium due to frequent scattering with particles from the radiation fluid. After the QCD transition (at $\sim 160 \mathrm{MeV}$ ) neutralino-lepton scattering is the most important process. The neutralinos decouple kinetically once the relaxation time $\tau$ becomes comparable with the Hubble time $t_{\mathrm{H}} \equiv H^{-1}$, which happens, depending on the parameters of the MSSM between $10 \mathrm{MeV}$ and a few $100 \mathrm{MeV}$. Once collisions of neutralinos with particles from the radiation fluid cease, the equation of state becomes nonrelativistic $(P \approx 0)$ and neutralino matter starts its life as cold dark matter [9, 10, 12]. 
In the present work we calculate the temperatures of kinetic decoupling and last scattering of neutralino CDM for the case of a bino-like neutralino. A first estimate of the kinetic decoupling temperature, based on dimensional arguments, was given by Schmid, Schwarz, and Widerin [9], which has been confirmed recently by more detailed calculations by Chen, Kamionkowski, and Zhang [12]. However, the authors of Ref. [12] ignored the fact that the relevant time scale that has to be compared to the Hubble time is the relaxation time, rather than the collision time. It was shown explicitly that photonneutralino scattering is suppressed by several orders of magnitudes, compared to lepton-neutralino scattering.

During the process of kinetic decoupling, collisional damping can smear out primordial fluctuations in neutralino CDM below some mass scale $M_{\mathrm{d}}$. Free streaming gives rise to additional damping below $M_{\mathrm{fs}}(t)$, which depends, in contrast to $M_{\mathrm{d}}$, on time. Both damping mechanisms together give rise to a sharp cut-off in the primordial power spectrum of neutralino CDM, that typically lies at $M \sim 10^{-7} M_{\odot}$ at the time of matter-radiation equality. We have presented preliminary estimates in Ref. [10]. In Ref. [12] it was pointed out that the estimate of induced damping found in [1] , is wrong by several orders of magnitude mainly because the cross section for elastic scatterings of photons with neutralinos has been overestimated.

We also show that bulk viscosity, besides shear viscosity, can not be neglected (as has been done in [11]) in the situation when a nonrelativistic component decouples from a radiation fluid. At first sight this is a surprising result since bulk viscosity usually goes along with the transfer of energy to internal degrees of freedom or with particle production. None of these mechanisms is available here. However, CDM and radiation have to be treated as two separate fluids and the bulk viscosity of the CDM fluid just reflects the energy dissipation from the CDM fluid to the radiation fluid, which is however a negligible effect for the radiation fluid since the energy density of the CDM fluid is tiny compared to the energy density of the radiation fluid at kinetic decoupling. On the other hand the heat conduction (which has been considered in [11]) can be neglected for the CDM fluid. The reason is simple, the neutralinos are too slow.

The paper is organized as follows: A short summary of mass limits and our assumptions about the lightest neutralino is given in Sec. II. Then we review the simplest calculation of chemical decoupling for pedagogical reasons and compare that with our detailed calculation of the kinetic decoupling and last scattering temperatures (Sec. III). In the following section (IV) we introduce CDM as an imperfect fluid, along the lines described in 13 15. The kinetic theory for the description of CDM is explained in Sec. V, and the coefficients of transport are calculated in Sec. VI. For this purpose we generalize the program by Weinberg [15] and Straumann [16] to the situation of a nonrelativistic component that decouples from a relativistic fluid [Weinberg and Straumann treat the problem of decoupling of a relativistic component (photons) from a nonrelativistic fluid (baryons)]. This finally allows us to calculate the damping scale from kinetic decoupling (Sec. VII) and free streaming (Sec. VIII). We conclude with a short discussion of the implications of our findings. The relevant cross sections are calculated in App. A and some useful thermodynamical relations can be found in App. B.

\section{NEUTRALINOS}

A direct lower limit for the neutralino mass $M_{\tilde{\chi}}$ is provided by the LEP experiments, $M_{\tilde{\chi}}>37 \mathrm{GeV}$ for any $\tan \beta$ and sfermion mass [17]. Reasonable assumptions (universal soft supersymmetry-breaking scalar masses at some higher scale) within the MSSM (constrained MSSM) and taking results of the Higgs searches into account raises the lower limit to about $50 \mathrm{GeV}$ [17,18. Incorporating also constraints from $b \rightarrow s \gamma$ decays and assuming that neutralino dark matter is cosmologically interesting $\left(0.1<\omega_{\tilde{\chi}}<0.3\right)$ a lower limit as high as $M_{\tilde{\chi}} \geq 140 \mathrm{GeV}$ can be derived 18]. The cosmological upper limit also gives rise to an upper limit on the neutralino mass. It is essential to include the effects of the next-to-lightest supersymmetric particles and coannihilations, as well as the contribution from poles and thresholds properly 18 20]. A detailed analysis gives $M_{\tilde{\chi}}<600$ $\mathrm{GeV} 18$. Since many untested, although reasonable, assumptions go into these limits, we decided for this work to assume $M_{\tilde{\chi}}>50 \mathrm{GeV}$.

The neutralinos are linear combinations of the neutral gauginos and the two higgsinos of the theory, i. e.,

$$
\tilde{\chi}_{1}^{0}=Z_{11} \tilde{B}^{0}+Z_{12} \tilde{W}_{3}^{0}+Z_{13} \tilde{H}_{1}^{0}+Z_{14} \tilde{H}_{2}^{0}
$$

expressed in terms of mass eigenstates. The $Z_{1 j}, j \in$ $\{1,2,3,4\}$ are elements of a real orthogonal matrix which diagonalizes the neutralino mass matrix. In most of the parameter space of the constrained MSSM the LSP is a $\tilde{B}^{0}$. We assume $\tilde{\chi}_{1}^{0} \equiv \tilde{\chi} \approx \tilde{B}^{0}$.

For a pure bino the interaction with a standard model fermion $F$ is given via the exchange of the related leftor right-handed sfermion $\tilde{F}_{L, R}$ as follows:

$$
\mathcal{L}_{F \tilde{F} \tilde{\chi}}=-\sqrt{2} g \bar{F}\left\{b_{F} \tilde{F}_{L} \mathcal{P}_{R}-c_{F} \tilde{F}_{R} \mathcal{P}_{L}\right\}+\text { h.c. }
$$

where $g$ is the electroweak coupling constant, $\mathcal{P}_{L, R}$ denotes the left and right chiral projection operator. The left and right chiral vertices are given by

$$
\begin{aligned}
& b_{F}=Z_{11} \frac{Y_{F}}{2} \tan \theta_{\mathrm{W}}+\mathrm{Z}_{12} \mathrm{~T}_{3 \mathrm{~F}}, \\
& c_{F}=Z_{11} Q_{F} \tan \theta_{\mathrm{W}} .
\end{aligned}
$$

Here $Y_{F}, T_{3 F}$ and $Q_{F}$ are the weak hypercharge, isospin and electrical charge of the involved fermions. 


\section{CHEMICAL AND KINETIC DECOUPLING}

There is a large difference between the temperature of chemical decoupling, $T_{\mathrm{cd}}$, and the temperature of kinetic decoupling, $T_{\mathrm{kd}}$, of neutralino cold dark matter. This is a characteristic feature of weakly interacting massive particles (WIMPs). Chemical decoupling (freeze-out) fixes the relic abundance of neutralinos and therefore the present value of $\Omega_{\mathrm{CDM}} h^{2}$. Before kinetic decoupling the neutralinos are tightly coupled to radiation, after kinetic decoupling the neutralinos acquire the properties of CDM. Namely, the neutralinos interact with radiation only via gravity and their pressure is negligible compared to their energy density well before matter-radiation equality.

Let us first review the process of chemical decoupling, which is a useful warm up for the kinetic decoupling that is explained subsequently. We assume that the neutralino is the bino, which reduces the number of free parameters to the bino mass, $M_{\tilde{\chi}}$ and to the universal sfermion mass, $M_{\tilde{F}}$. For a more complete picture including higgsino admixture, thresholds, poles, and coannihilations see [21,19,20.

At $T \gg T_{\text {cd }}$ neutralinos are kept in chemical equilibrium with all standard model fermions $F$ in the heat bath at temperature $T$ via annihilation processes $\tilde{\chi}+\tilde{\chi} \leftrightarrow$ $F+\bar{F}$. From (2) one can calculate the annihilation rate for $\tilde{\chi}+\tilde{\chi} \rightarrow F+\bar{F}$ [21,

$$
\begin{aligned}
\Gamma_{\mathrm{ann}}(T) & =\sum_{F}\left\langle v \sigma_{\mathrm{ann}}\right\rangle(T) n_{\tilde{\chi}}(T) \\
& =\frac{2}{\pi} \sum_{F}\left(\frac{G_{\mathrm{F}} M_{W}^{2}}{M_{\tilde{F}}^{2}+M_{\tilde{\chi}}^{2}}\right)^{2}\left[\left(b_{F}^{2}+c_{F}^{2}\right)^{2} m_{F}^{2}+\right. \\
& \left.+4\left(b_{F}^{4}+c_{F}^{4}\right) \frac{M_{\tilde{F}}^{4}+M_{\tilde{\chi}}^{4}}{\left(M_{\tilde{F}}^{2}+M_{\tilde{\chi}}^{2}\right)^{2}} M_{\tilde{\chi}} T\right] n_{\tilde{\chi}}(T)
\end{aligned}
$$

Here, $\langle\ldots\rangle$ denotes thermal averaging and $v$ is the Moeller-velocity. In order to obtain (6) we expanded $v \sigma_{\text {ann }}$ for small $m_{F} / M_{\tilde{\chi}}$ and small $v$. More details can be found in appendix A. Note that the first term in the square brackets contributes practically only for top quarks $(F=t)$. However, we will assume below that $M_{\tilde{\chi}}<m_{t}$ such that the second term will be dominant in our estimate. We neglect annihilation of neutralinos into final states containing gauge and Higgs bosons, such as $\tilde{\chi} \tilde{\chi} \leftrightarrow\{W W, Z Z, H H, H W, H Z\}$, since these channels are particularly important for Higgsino-like and mixedstate neutralinos, but are subdominant when compared to the fermion-antifermion channels in the case that the neutralino is mostly a gaugino [19]. Since we restrict our attention to a pure bino, there is no contribution from diagrams with $Z^{0}$ exchange at tree level and therefore the $Z^{0}$ pole does not invalidate our estimate below.

As the universe expands the temperature eventually falls below the neutralino mass $M_{\tilde{\chi}}$ and the number density $n_{\tilde{\chi}}$ of neutralinos decreases exponentially. Once the annihilation rate $\Gamma_{\text {ann }}$ becomes comparable to the expansion rate $H$ of the universe neutralinos no longer find other neutralinos to annihilate. We use the condition $\Gamma_{\text {ann }}=H$ to define the temperature of chemical decoupling $T_{\mathrm{cd}}$. Solving this equation iteratively yields $\left(x \equiv M_{\tilde{\chi}} / T\right)$

$$
\begin{aligned}
& x_{\mathrm{cd}}^{(0)}=\ln \left[1.6 \times 10^{-4} \frac{M_{\mathrm{Pl}}\left(M_{\tilde{F}}^{4}+M_{\tilde{\chi}}^{4}\right) M_{\tilde{\chi}}^{3}}{\left(M_{\tilde{F}}^{2}+M_{\tilde{\chi}}^{2}\right)^{4}}\right], \\
& x_{\mathrm{cd}}^{(1)} \approx x_{\mathrm{cd}}^{(0)}-\frac{1}{2} \ln x_{\mathrm{cd}}^{(0)},
\end{aligned}
$$

as long as the bino mass is well below the top mass, but large compared to the bottom mass. In deriving (7) we assumed equal masses for all sfermions. Exploring the parameter space of the MSSM we typically find $x_{\mathrm{cd}} \approx 25$, cf. figure 1.

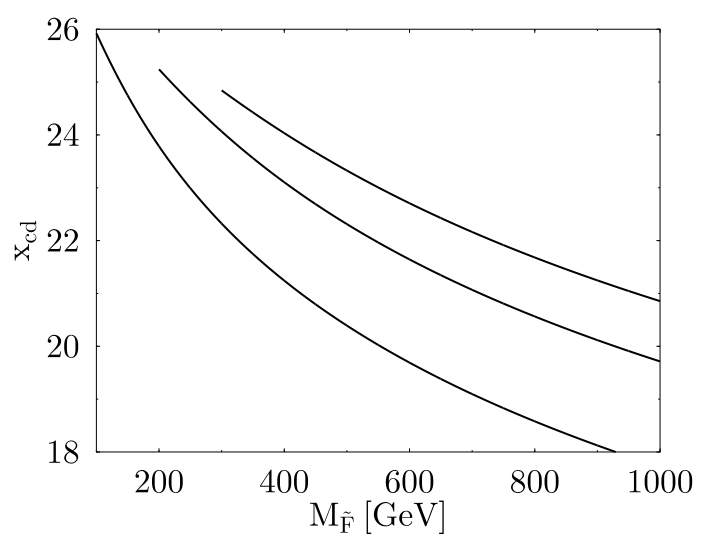

FIG. 1. The chemical decoupling as a function of the sfermion mass $M_{\tilde{F}}$ for three values of the neutralino mass $M_{\tilde{\chi}}=50,100,150 \mathrm{GeV}$ (increasing from bottom to top).

The relic abundance of neutralinos is now easily obtained as $n_{\tilde{\chi}}\left(T_{0}\right)=n_{\tilde{\chi}}\left(T_{\mathrm{cd}}\right) s\left(T_{0}\right) / s\left(T_{\mathrm{cd}}\right)$, where $T_{0}=$ $2.725 \mathrm{~K}$ and $s$ denotes the entropy density of the universe. It is a good approximation to use the equilibrium distribution for the number density at $T_{\text {cd }}$, although in a more advanced treatment the corresponding kinetic equation should be solved. From the number density $n_{\tilde{\chi}}\left(T_{0}\right)$ we may easily compute $\omega_{\tilde{\chi}} \equiv \Omega_{\tilde{\chi}} h^{2}$, which is plotted in Fig. 2 as a function of $M_{\tilde{\chi}}$ for typical values of the sfermion mass.

Below $T_{\text {cd }}$ the neutralinos are kept in local thermal equilibrium via elastic scattering processes $\tilde{\chi}+F \rightarrow \tilde{\chi}+$ $F$. After the QCD phase transition only leptons $L$ remain as scattering partners for the neutralinos. We neglect scatterings with pions, which is important for $T>m_{\pi}$ only. It will turn out that in most cases $T_{\mathrm{kd}} \ll m_{\pi}$. Scattering with nucleons is not important due to the tiny number density of baryons. From (2) one can calculate the rate of elastic scatterings $\tilde{\chi}+L \rightarrow \tilde{\chi}+L$ 21]. We find

$\Gamma_{\mathrm{el}}=\sum_{L}\left\langle v \sigma_{\mathrm{el}}\left(E_{L}\right)\right\rangle(T) n_{L}(T)$ 


$$
=\frac{288}{\pi} \sum_{L}\left(b_{L}^{4}+c_{L}^{4}\right)\left(\frac{G_{\mathrm{F}} M_{W}^{2}}{M_{\tilde{L}}^{2}-M_{\tilde{\chi}}{ }^{2}}\right)^{2} T^{2} n_{L}(T) .
$$

$E_{L}$ denotes the energy and $n_{L}$ the number density of the leptons. In deriving (9) we approximate the Mandelstam variable $s \approx M_{\tilde{\chi}}^{2}+2 M_{\tilde{\chi}} E_{L}$. Note that the Moeller velocity in this case is $v \approx 1$ to a very good approximation.

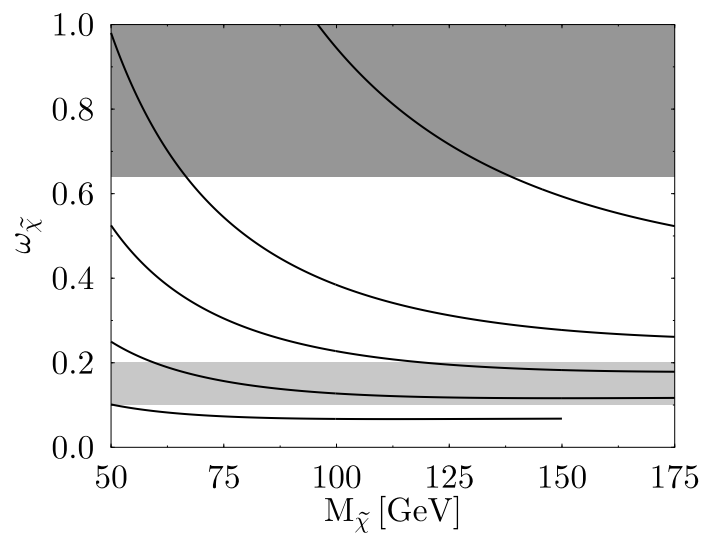

FIG. 2. The relic abundance of neutralinos expressed by $\omega_{\tilde{\chi}}=\Omega_{\tilde{\chi}} h^{2}$ as a function of the neutralino mass $M_{\tilde{\chi}}$ for different values of the sfermion mass $M_{\tilde{F}}=150,200,250,300,400$ $\mathrm{GeV}$. The sfermion mass increases from the bottom to the top. The dark shaded region is excluded by the conservative assumptions: $\Omega \leq 1$ and $h<0.8$. The light shaded region indicates typical values of $\omega$ in a $\Lambda$ CDM model.

In analogy to the chemical decoupling the condition $\Gamma_{\mathrm{el}}=H$ defines the temperature at the time the last elastic interactions between neutralinos and the rest of the Universe take place. This last scattering temperature is given by

$$
T_{\mathrm{ls}}=\left[8.7 \times 10^{-3} \frac{m_{\mathrm{Pl}}}{\left(M_{\tilde{L}}^{2}-M_{\tilde{\chi}}^{2}\right)^{2}}\right]^{-1 / 3} .
$$

Typical values are $1 \mathrm{MeV}$ to $10 \mathrm{MeV}$, e.g., $T_{\mathrm{ls}}=2.3(2.5)$ $\mathrm{MeV}$ for $M_{\tilde{\chi}}=100(150) \mathrm{GeV}$ and $M_{\tilde{L}}=200(250) \mathrm{GeV}$. However, this is not the temperature at which neutralinos decouple kinetically. The kinetic decoupling temperature is defined through the relaxation time $\tau$, rather than by the collision time $\tau_{\text {coll }}=1 / \Gamma_{\text {el }}$. This can be easily understood by the following argument.

The relaxation time $\tau$, i.e., the time neutralinos need to return to local thermal equilibrium after a deviation from it, can be estimated from the typical number of scatterings that is needed to change the momentum of the neutralino significantly. The typical momentum transfer in a single elastic scattering event is tiny compared to the average momentum of the neutralinos. This is easily seen from the averaged Mandelstam variable $t$,

$$
\left(\Delta p_{\tilde{\chi}}\right)^{2} \equiv-\frac{1}{\sigma_{\mathrm{el}}} \int \frac{\mathrm{d} \sigma_{\mathrm{el}}}{\mathrm{d} t} t \mathrm{~d} t=2 E_{L}^{2}
$$

The leptons are kept in local thermal equilibrium through the frequent interactions among themselves and the equipartition theorem gives $E_{L}=3 / 2 T$. Comparing the rms momentum transfer with the typical neutralino momentum $p_{\tilde{\chi}}$ we find $\Delta p_{\tilde{\chi}} / p_{\tilde{\chi}}=\sqrt{3 / 2} T / M_{\tilde{\chi}} \ll 1$. This means that a huge number $N(T)$ of elastic scatterings is needed to keep or to establish thermal equilibrium, $N(T)=p_{\tilde{\chi}} / \Delta p_{\tilde{\chi}}=\sqrt{3 / 2} M_{\tilde{\chi}} / T$. We can now estimate the relaxation time as

$$
\tau(T) \approx \sqrt{\frac{2}{3}} \frac{M_{\tilde{\chi}}}{T} \tau_{\text {coll }}
$$

Note that $\tau(T) \sim 1 / T^{6}$.

The kinetic decoupling of the neutralinos happens when the relaxation time $\tau$ becomes comparable to the Hubble time $1 / H$. We denote the corresponding temperature by $T_{\mathrm{kd}}$, which is given by

$$
T_{\mathrm{kd}}=\left[1.2 \times 10^{-2} \frac{m_{\mathrm{Pl}}}{M_{\tilde{\chi}}\left(M_{\tilde{L}}^{2}-M_{\tilde{\chi}}^{2}\right)^{2}}\right]^{-1 / 4},
$$

where we assumed that all leptons except the tau are relativistic, but we neglected the contribution of pions which are important at temperatures of about $130 \mathrm{MeV}$. Above the QCD phase transition at about $160 \mathrm{MeV}$ much more interaction partners are available and our formula should be modified. Exploring the parameter space of the MSSM we typically find that $T_{\mathrm{kd}}$ is of the order $10 \mathrm{MeV}$ to $100 \mathrm{MeV}$, cf. figure 3. For $M_{\tilde{\chi}}=100(150) \mathrm{GeV}$ and $M_{\tilde{L}}=200(250) \mathrm{GeV}$ we find $T_{\mathrm{kd}}=28(36) \mathrm{MeV}$, whereas the chemical decoupling for the same set of parameters happens at $T_{\mathrm{cd}}=4.0(5.9) \mathrm{GeV}$, a difference of more than two orders of magnitude.

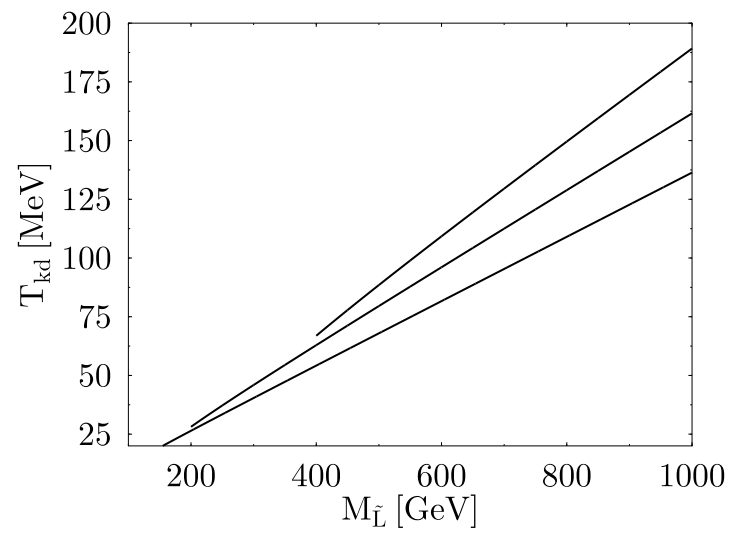

FIG. 3. The temperature of kinetic decoupling of neutralinos from radiation as a function of the sfermion mass for $M_{\tilde{\chi}}=50,100,200 \mathrm{GeV}$ (bottom to top).

The large difference between $T_{\mathrm{cd}}$ and $T_{\mathrm{kd}}$ is mainly due to the different target densities in the annihilation [Eq. (5)] and elastic scattering rates [Eq. (8)]. For annihilations the target density is given by the number density $n_{\tilde{\chi}}$ of neutralinos in the universe. The number density 
of neutralinos is suppressed by the Boltzmann factor at chemical decoupling. In contrast the target density for elastic scattering processes is given by the number density of all relativistic leptons.

During the cooling from $T_{\mathrm{cd}}$ to $T_{\mathrm{kd}}$ the leptons behave as a perfect radiation fluid which tries to keep the neutralinos in thermal equilibrium through elastic scattering processes. The neutralinos on the other hand may be described as a nonrelativistic, imperfect fluid.

\section{CDM AS A FLUID}

For temperatures $T>T_{\text {cd }}$ the particle content of the universe may be described by a single radiation fluid which is in local thermal equilibrium. For temperatures $T_{\mathrm{kd}}<T<T_{\mathrm{cd}}$ the radiation fluid is tightly coupled to the CDM fluid. Hence, both fluids have the same temperature and velocity four-vectors. Around $T_{\mathrm{kd}}$ the CDM fluid starts to decouple kinetically from the radiation fluid and becomes an imperfect fluid. The departure from local thermal equilibrium is generated by dissipation, i.e. by shear and bulk viscosity (we show below that the coefficient of heat conduction vanishes). For temperatures $T<T_{\mathrm{kd}}$ both fluids are decoupled and the CDM fluid is freely streaming. Since $\Omega_{\mathrm{CDM}}=\left(a / a_{\mathrm{eq}}\right) \Omega_{\mathrm{rad}} \ll \Omega_{\mathrm{rad}}$ for $T \gg T_{\mathrm{eq}}$, the radiation fluid remains in local thermal equilibrium throughout the decoupling process.

The current density and the energy-momentum tensor of the radiation fluid $(\mathrm{R})$ are given by

$$
\begin{aligned}
J_{\mathrm{R}}^{\mu} & =n_{\mathrm{R}} V^{\mu}, \\
T_{\mathrm{R}}^{\mu \nu} & =\rho_{\mathrm{R}} V^{\mu} V^{\nu}-P_{\mathrm{R}} h^{\mu \nu} .
\end{aligned}
$$

Here, $n_{\mathrm{R}}, \rho_{\mathrm{R}}$, and $P_{\mathrm{R}}$ are the number density, the energy density, and the pressure of the radiation fluid respectively. $V$ is the velocity four-vector with $V^{2}=1$. $h^{\mu \nu}=g^{\mu \nu}-V^{\mu} V^{\nu}$ is the projection operator on the plane perpendicular to $V$. The radiation fluid variables only depend on the temperature of radiation, $T_{\mathrm{R}}$, since there are no relevant conserved quantum numbers besides $\mathrm{R}$-parity, which is taken into account in the CDM fluid.

The current density and the energy-momentum tensor of the imperfect CDM fluid can be written as 13.15

$$
\begin{aligned}
J^{\mu} & =n U^{\mu}+J^{(1) \mu}, \\
T^{\mu \nu} & =\rho(T, n) U^{\mu} U^{\nu}-P(T, n) h^{\mu \nu}+T^{(1) \mu \nu .} .
\end{aligned}
$$

$n, U, \rho(T, n)$, and $P(T, n)$ are the number density, fourvelocity, energy density, and pressure of the CDM fluid, respectively. We omit the subscript $\tilde{\chi}$ for the CDM component in Secs. IV, V, and VI, since the results of these sections hold true for more general forms of WIMP CDM. The projection $h$ is orthogonal to $U$ here. We do not introduce two different symbols in the following, because it is always clear from the context to which velocity $h$ is referring. For the CDM fluid $T$ and $n$ are independent variables, since R-parity, i.e., the number of neutralinos, is conserved. $T$ is not necessarily identical to $T_{\mathrm{R}}$, although this is the case when both fluids are in thermal equilibrium. In the adiabatic limit all space-time gradients are negligible, i.e., $J^{(1)}=0, T^{(1)}=0$, and the CDM fluid has the same temperature and the same velocity as the radiation fluid, $U=V$.

For an imperfect fluid described by (16) and (17) number density, energy density and velocity are not defined uniquely. To fix this ambiguity we define the number and energy density by

$$
\begin{aligned}
n & \equiv U_{\mu} J^{\mu}, \\
\rho(T, n) & \equiv U_{\mu} U_{\nu} T^{\mu \nu},
\end{aligned}
$$

such that the hydrodynamic and thermodynamic definitions of $n$ and $\rho$ coincide. The velocity is fixed to be the comoving velocity of the CDM particles

$$
U^{\mu} \equiv\left(J_{\lambda} J^{\lambda}\right)^{-\frac{1}{2}} J^{\mu}
$$

This choice of the velocity corresponds to the one of Eckart [13], and was applied to relativistic fluids by Weinberg 15. An alternative would be the choice of Landau [14], where the velocity is fixed to coincide with the velocity of the energy-momentum flow. In other words (18) requires $J^{(1)}$ to be perpendicular to $U$. In the same sense (19) requires $T^{(1)}$ to project on the plane perpendicular to $U$. Condition (20) means that $J^{(1)}$ has to vanish. With this choice

$$
\begin{aligned}
J^{\mu} & =n U^{\mu}, \\
T^{\mu \nu} & =\rho(T, n) U^{\mu} U^{\nu}-P(T, n) h^{\mu \nu}+T^{(1) \mu \nu} .
\end{aligned}
$$

The construction of $T^{(1)}$ from a first-order formalism is given in [15]. The starting point is that $T^{(1)}$ has to be expressed in the equilibrium variables and their gradients. The basic observation is that the variation of the entropy per particle, $\sigma$, along the adiabatic flow is generated by the change of $T^{(1)}$ in the same space-time direction, i.e.,

$$
n T \dot{\sigma}=-U_{\mu} T_{, \nu}^{(1) \mu \nu},
$$

where the dot denotes the hydrodynamic derivative, $\dot{()} \equiv$ $U^{\mu}()_{, \mu}$. Equivalently we may write for the entropy current four-vector $S^{\mu} \equiv n \sigma U^{\mu}+1 / T U_{\lambda} T^{(1) \lambda \mu}$ (as can be easily seen in the comoving frame, $S^{0}=n \sigma=s$ is indeed the entropy density and $T S^{i}=T^{(1) 0 i}$ is the nonadiabatic contribution to the energy-momentum flow, which is the heat flow)

$$
T^{2} S_{, \lambda}^{\lambda}=-\left(U_{\mu} T_{, \nu}-T U_{\mu, \nu}\right) T^{(1) \mu \nu} .
$$

As a consequence only space-time derivatives of $T$ and $U$ can occur in $T^{(1)}$ in order to keep the rate of entropy production positive for all fluid configurations.

The perturbed energy-momentum tensor may be expressed in terms of the heat-flow vector

$$
Q_{\mu}=T_{, \mu}-T \dot{U}_{\mu}
$$


and the traceless shear tensor

$$
W_{\mu \nu}=U_{\mu, \nu}+U_{\nu, \mu}-\frac{2}{3} g_{\mu \nu} U_{, \lambda}^{\lambda} .
$$

With these abbreviations we write 15

$$
\begin{aligned}
T^{(1) \mu \nu}= & \zeta h^{\mu \nu} U_{, \lambda}^{\lambda}+\eta h^{\mu \rho} h^{\nu \sigma} W_{\rho \sigma}- \\
& \chi\left(h^{\lambda \mu} U^{\nu}+h^{\lambda \nu} U^{\mu}\right) Q_{\lambda},
\end{aligned}
$$

where $\zeta, \eta$ and $\chi$ are the coefficients for bulk viscosity, shear viscosity and heat conduction.

These parameters need to be calculated in the framework of a non-equilibrium theory.

\section{KINETIC DESCRIPTION OF CDM}

In this and the following section we generalize a method by Straumann [16] to calculate the coefficients of transport for a species of massive particles that decouple kinetically. In Ref. [16] the problem of the decoupling of radiation quanta was treated.

Let $F(p, x)$ be the distribution function of neutralinos. $F(p, x)$ is normalized in such a way that $F(p, x) \mathrm{d}^{3} p \mathrm{~d}^{3} x$ gives the number of quanta in the volume $\mathrm{d}^{3} x$ centered at the space-time point $x$ and three-momentum within $\mathrm{d}^{3} p$. We assume that the neutralinos are close to thermal equilibrium and make the ansatz

$$
F=F^{(0)}+F^{(1)} \quad \text { with } \quad\left|F^{(1)}\right| \ll F^{(0)},
$$

where

$$
F^{(0)}(p, x)=\frac{g}{(2 \pi)^{3}} \frac{1}{\exp \left(\frac{p \cdot V}{T_{\mathrm{R}}}-\alpha\right) \pm 1} .
$$

Here, $T_{\mathrm{R}}$ is the local temperature of the radiation fluid and $\alpha$ is the local pseudo chemical potential of the neutralino. To first order in the collision time, $F^{(1)}$ is a solution of the kinetic equation

$$
(p \cdot \partial) F^{(0)}=L\left[F^{(1)}\right] \text {. }
$$

$L$ is supposed to be a linear functional in $F^{(1)}$. In the linear regime one often uses $L\left[F^{(1)}\right]=-\omega \tau^{-1} F^{(1)}$ as a realistic model for the collision integral.

In consideration of Eckarts approach to the hydrodynamics of imperfect fluids [13] we introduce a four-vector perpendicular to $V$,

$$
n^{\mu}=|\vec{p}|^{-1}\left(p^{\mu}-\omega V^{\mu}\right)
$$

with $\omega=p \cdot V$ such that $F^{(1)}$ can be considered as a function of $\omega, n$ and $x$ or equivalent as a function of the projection of $p$ in the direction of $V$ and perpendicular to it.

Following 22] we may now expand $F_{P}^{(1)}(\omega, n, x)$ into polynomials in $n$

$$
\begin{aligned}
F^{(1)}(\omega, n, x)= & A(\omega, x)+B_{\mu}(\omega, x) n^{\mu}+ \\
& C_{\mu \nu}(\omega, x)\left(n^{\mu} n^{\nu}+\frac{1}{3} h^{\mu \nu}\right)+\ldots
\end{aligned}
$$

It is clear from the kinetic equation 30 that we need to know how the functional $L$ operates on $F^{(1)}$. In order to solve this problem we note that $F$ is defined to be invariant under Lorentz-transformations. Let $G_{x}$ be the group of all Lorentz-transformations leaving $V(x)$ invariant at every space-time point $x$, i.e. $G_{x}$ is the little group with respect to $V . G_{x}$ is isomorphic to the Lie-group $S O(3)$. Since $F$ is invariant under $G_{x}$ at every space-time point $x, F^{(1)}$ is invariant and $(32)$ is an expansion into irreducible polynomials with respect to $G_{x}$. From equation (30) it follows that the linear functional $L$ is a scalar with respect to $G_{x}$. Therefore it operates on the irreducible subspace spanned by the polynomials in (32) as a multiple of the identity. Thus we can write

$$
\begin{aligned}
L\left[F^{(1)}\right]= & -\omega\left[\kappa_{0} A+\kappa_{1} B_{\mu} n^{\mu}+\right. \\
& \left.+\kappa_{2} C_{\mu \nu}\left(n^{\mu} n^{\nu}+\frac{1}{3} h^{\mu \nu}\right)\right]+\ldots,
\end{aligned}
$$

where the $\kappa_{j}(j \in\{0,1,2\})$ are functions of $\omega$ and $x$ only. Note that in the case of the model for the collision integral discussed above $\kappa_{j}=\tau^{-1}$ for all $j \in\{1,2,3\}$.

Next we derive expressions for $A, B_{\mu}$ and $C_{\mu \nu}$ in terms of $V, T_{\mathrm{R}}, \alpha$ and $\kappa_{j}$ using the kinetic equation (30). In order to do this we have to define a measure $\mathrm{d} \Omega_{V}$ on the two dimensional surface $S=\left\{p: p^{2}=M^{2}, p^{0}>0\right.$ and $\omega=$ const. $\}$. $d \Omega_{V}$ is normalized such that

$$
\frac{1}{4 \pi} \int_{S} \mathrm{~d} \Omega_{V}=1
$$

The irreducible polynomials in (30) are orthogonal with respect to $\mathrm{d} \Omega_{V}$ and are normalized as follows

$$
\begin{aligned}
& \frac{1}{4 \pi} \int_{S} \mathrm{~d} \Omega_{V} n^{\mu} n^{\nu}=-\frac{1}{3} h^{\mu \nu}, \\
& \frac{1}{4 \pi} \int_{S} \mathrm{~d} \Omega_{V}\left(n^{\mu} n^{\nu}+\frac{1}{3} h^{\mu \nu}\right)\left(n^{\alpha} n^{\beta}+\frac{1}{3} h^{\alpha \beta}\right)= \\
& \quad \frac{1}{15} h^{\{\mu \nu} h^{\alpha \beta\}}-\frac{1}{9} h^{\mu \nu} h^{\alpha \beta} .
\end{aligned}
$$

Now it is possible to project out every tensor in the expansion of $L\left[F^{(1)}\right.$, equation (32). Taking moments of (30) and using (32) we obtain

$$
\begin{aligned}
A & =\frac{1}{\kappa_{0}} \frac{\omega \Phi^{\prime}}{T_{\mathrm{R}}}\left[\frac{\dot{T}_{\mathrm{R}}}{T_{\mathrm{R}}}+\frac{1}{3}\left(1-\frac{m^{2}}{\omega^{2}}\right)^{2} V_{, \lambda}^{\lambda}+\frac{T_{\mathrm{R}}}{\omega} \dot{\alpha}\right], \\
B_{\mu} & =\frac{1}{\kappa_{1}} \frac{\omega \Phi^{\prime}}{T_{\mathrm{R}}}\left(1-\frac{m^{2}}{\omega^{2}}\right)^{\frac{1}{2}} h_{\mu}^{\lambda}\left[\frac{1}{T_{\mathrm{R}}} Q_{\lambda}+\frac{T_{\mathrm{R}}}{\omega} \alpha, \lambda\right], \\
C_{\mu \nu} & =-\frac{1}{\kappa_{2}} \frac{\omega \Phi^{\prime}}{T_{\mathrm{R}}} \frac{1}{2}\left(1-\frac{m^{2}}{\omega^{2}}\right)^{2} h_{\mu}^{\lambda} h_{\nu}^{\gamma} W_{\lambda \gamma} .
\end{aligned}
$$


Here, $\Phi^{\prime}\left(\omega / T_{\mathrm{R}}-\alpha\right)$ denotes the external derivative of $F^{(0)}(\omega, x)$. In calculating the integrals we replaced $(p$. д) $T_{\mathrm{R}}$ with $\omega(V \cdot \partial) T_{\mathrm{R}}$ and $p_{\beta}(p \cdot \partial) V^{\beta}$ with $|\vec{p}|^{2} n_{\beta}(n$. д) $V^{\beta}$.

The coefficients (37) and (38) depend on the variations of $T_{\mathrm{R}}$ and $\alpha$ along the adiabatic flow and the directional derivative of $\alpha$ in the plane perpendicular to the adiabatic flow. In order to make sure that the rate of entropy production along the adiabatic flow is positive for all kinematical configurations these derivatives need to be proportional to space-time gradients of $T_{\mathrm{R}}$ and $V$. Using the adiabatic relations derived in Appendix B we find

$$
\begin{aligned}
A= & -\frac{1}{\kappa_{0}} \frac{\omega \Phi^{\prime}}{T_{\mathrm{R}}} \times \\
& {\left[\left(\frac{\partial P}{\partial \rho}\right)_{n}-\frac{1}{3}\left(1-\frac{m^{2}}{\omega^{2}}\right)^{2}+\omega^{-1}\left(\frac{\partial P}{\partial n}\right)_{\rho}\right] V_{, \lambda}^{\lambda}, } \\
B_{\mu}= & \frac{1}{\kappa_{1}} \frac{\omega \Phi^{\prime}}{T_{\mathrm{R}}}\left(1-\frac{m^{2}}{\omega^{2}}\right)^{\frac{1}{2}}\left[\frac{\omega}{T_{\mathrm{R}}}-\frac{w}{n T_{\mathrm{R}}}\right] h_{\mu}^{\lambda} Q_{\lambda}, \\
C_{\mu \nu}= & -\frac{1}{\kappa_{2}} \frac{\omega \Phi^{\prime}}{T_{\mathrm{R}}} \frac{1}{2}\left(1-\frac{m^{2}}{\omega^{2}}\right)^{2} h_{\mu}^{\lambda} h_{\nu}^{\gamma} W_{\lambda \gamma},
\end{aligned}
$$

with the enthalpy $w=\rho+P$.

\section{A. Current density}

In kinetic theory the current density of neutralinos is given by

$$
J^{k \mu}=\int \frac{\mathrm{d}^{3} p}{p^{0}} p^{\mu} F(\omega, n, x) .
$$

Considering our ansatz (28), we may write

$$
J^{k \mu}=J^{k(0) \mu}+J^{k(1) \mu},
$$

with the definitions

$$
\begin{aligned}
J^{k(0) \mu} & \equiv \int \frac{\mathrm{d}^{3} p}{p_{0}} p^{\mu} F^{(0)}(\omega, x)=n^{k} V^{\mu}, \\
n^{k} & =4 \pi \int_{M}^{\infty} \mathrm{d} \omega\left(\omega^{2}-M^{2}\right)^{1 / 2} \omega \Phi
\end{aligned}
$$

and

$$
J^{k(1) \mu} \equiv \int \frac{\mathrm{d}^{3} p}{p_{0}} p^{\mu} F^{(1)}(\omega, n, x)=\Delta n V^{\mu}+J_{\text {diff }}^{\mu}
$$

$\Delta n$ is generated by the coefficient $A$ whereas $J_{\text {diff }}$ is generated by $B$ :

$$
\begin{aligned}
\Delta n & =4 \pi \int_{M}^{\infty} \mathrm{d} \omega\left(\omega^{2}-M^{2}\right)^{1 / 2} \omega A, \\
J_{\text {diff }}^{\mu} & =-\frac{4 \pi}{3} \int_{M}^{\infty} \mathrm{d} \omega\left(\omega^{2}-M^{2}\right) B^{\mu} .
\end{aligned}
$$

Let us rewrite the above expressions with help of the following notation:

$$
f_{a}^{(i, j)}\left(T_{\mathrm{R}}, \alpha ; x\right) \equiv-4 \pi \int_{M}^{\infty} \mathrm{d} \omega\left(\omega^{2}-M^{2}\right)^{i / 2} \omega^{j} \frac{\Phi^{\prime}}{T_{\mathrm{R}} \kappa_{a}} .
$$

Note that the mass dimension of $f_{a}^{(i, j)}$ is simply $i+j$. In terms of these functions we obtain

$$
\begin{aligned}
& \Delta n= \\
& {\left[f_{0}^{(1,2)}\left(\frac{\partial P}{\partial \rho}\right)_{n}-f_{0}^{(3,0)} \frac{1}{3}+f_{0}^{(1,1)}\left(\frac{\partial P}{\partial n}\right)_{\rho}\right] V_{, \lambda}^{\lambda},} \\
& J_{\text {diff }}^{\mu}=\frac{1}{3 T_{\mathrm{R}}}\left[f_{1}^{(3,0)}-f_{1}^{(3,-1)} \frac{w}{n}\right] h^{\mu \lambda} Q_{\lambda} .
\end{aligned}
$$

\section{B. Energy-momentum tensor}

The energy-momentum tensor of neutralino CDM is given by

$$
T^{k \mu \nu}=\int \frac{\mathrm{d}^{3} p}{p_{0}} p^{\mu} p^{\nu} F(\omega, n, x) .
$$

Again, in consideration of our ansatz we may write

$$
T^{k \mu \nu}=T^{k(0) \mu \nu}+T^{k(1) \mu \nu},
$$

with the definitions

$$
\begin{aligned}
T^{k(0) \mu \nu} & \equiv \int \frac{\mathrm{d}^{3} p}{p_{0}} p^{\mu} p^{\nu} F^{(0)}(\omega, x) \\
& =\rho^{k} V^{\mu} V^{\nu}-P^{k} h^{\mu \nu}, \\
\rho^{k} & =4 \pi \int_{M}^{\infty} \mathrm{d} \omega\left(\omega^{2}-M^{2}\right)^{1 / 2} \omega^{2} \Phi, \\
P^{k} & =\frac{4 \pi}{3} \int_{M}^{\infty} \mathrm{d} \omega\left(\omega^{2}-M^{2}\right)^{3 / 2} \Phi,
\end{aligned}
$$

and

$$
T^{k(1) \mu \nu} \equiv T_{A}^{(1) \mu \nu}+T_{B}^{(1) \mu \nu}+T_{C}^{(1) \mu \nu}+\ldots
$$

The labels $A, B, C$ indicate which tensor in the expansion (32) gives rise to the extra contribution. From (32) and (40) - 42) we find

$$
\begin{aligned}
& T_{A}^{(1) \mu \nu}=\Delta \rho V^{\mu} V^{\nu}-\Delta P h^{\mu \nu} \\
& T_{B}^{(1) \mu \nu}=\frac{1}{3 T_{\mathrm{R}}}\left[f_{1}^{(3,1)}-f_{1}^{(3,0)} \frac{w}{n}\right] V^{\{\mu} h^{\nu\} \lambda} Q_{\lambda}, \\
& T_{C}^{(1) \mu \nu}=\frac{1}{15} f_{2}^{(5,-1)} h^{\mu \lambda} h^{\nu \gamma} W_{\lambda \gamma},
\end{aligned}
$$

with 
$\Delta \rho=$

$$
\begin{aligned}
& {\left[f_{0}^{(1,3)}\left(\frac{\partial P}{\partial \rho}\right)_{n}-f_{0}^{(3,1)} \frac{1}{3}+f_{0}^{(1,2)}\left(\frac{\partial P}{\partial n}\right)_{\rho}\right]_{, \lambda}^{\lambda},} \\
& \Delta P=
\end{aligned}
$$

$$
\frac{1}{3}\left[f_{0}^{(3,1)}\left(\frac{\partial P}{\partial \rho}\right)_{n}-f_{0}^{(5,-1)} \frac{1}{3}+f_{0}^{(3,0)}\left(\frac{\partial P}{\partial n}\right)_{\rho}\right] V_{, \lambda}^{\lambda} .
$$

\section{COEFFICIENTS OF TRANSPORT}

In the following we calculate the coefficients of bulk and shear viscosity and the coefficient of heat conduction for neutralino CDM starting from the kinetic description.

In Sec. III we have introduced Eckart's approach to describe imperfect fluids [13]. The number density and the energy density of the CDM fluid coincide with the corresponding quantities in the adiabatic limit, see Eqs. (18) and (19), and the velocity of the CDM fluid is fixed via the particle current, see Eq. (20). These definitions together with the required space-time symmetries and the second law of thermodynamics determine the most general structure of $J^{(1)}$ and $T^{(1)}$, see [15], as given in Eq. (27).

To compare the kinetic description from the previous section with the approach of Eckart it is necessary that the conditions (18)-(20) are fulfilled. Instead we find for the kinetic description

$$
\begin{aligned}
V_{\mu} J^{k \mu} & =n^{k}+\Delta n, \\
V_{\mu} V_{\nu} T^{k \mu \nu} & =\rho^{k}+\Delta \rho, \\
h^{\mu \lambda} J_{\lambda} & =J_{\text {diff }}^{\mu} .
\end{aligned}
$$

Due to the non-equilibrium dynamics, the kinetic number density and energy density do not coincide with Eckart's definitions, (64) and (65) are in conflict with (18) and (19). Equation (66) shows the existence of a diffusion current in the plane perpendicular to $V$. As a consequence the current density four-vector does not point to the space-time direction that is required by the approach of Eckart (20). In the following we consider the temperature and the number density to be the independent thermodynamical variables.

Let us first establish the link between the current in the kinetic and the hydrodynamic descriptions. As a first step we make a transformation of the velocity, such that the diffusion current vanishes,

$$
V \rightarrow U-\left(n^{k}+\Delta n\right)^{-1} J_{\text {diff }},
$$

which allows us to write

$$
J^{\mu}=\left(n^{k}+\Delta n\right) U^{\mu}=n U^{\mu},
$$

from comparison with (18).

Let us now turn to the energy-momentum tensor. The transformation (67) with $n=n^{k}+\Delta n$ generates an extra contribution to the heat conduction since

$$
\begin{aligned}
\rho^{k} V^{\mu} V^{\nu}= & \rho^{k} U^{\mu} U^{\nu}- \\
& \frac{\rho^{k}}{n} U^{\mu} J_{\text {diff }}^{\nu}-\frac{\rho^{k}}{n} J_{\text {diff }}^{\mu} U^{\nu}+\mathcal{O}\left(J_{\text {diff }}^{2}\right) .
\end{aligned}
$$

It remains to find the relation between the kinetic, 65), and the hydrodynamic, (19), definition of the energy density. The point is that the definitions of temperature in the approach of Eckart and in the kinetic theory under consideration are different [15]. In kinetic theory there is a unique way to define temperature as the temperature of the leptons and photons which stay in thermal equilibrium during and after the kinetic decoupling of the neutralinos. In the approach of Eckart the temperature was chosen such that the energy density agrees with the one in the adiabatic limit. Thus it is clear that the difference in the definitions should be generated by $\Delta \rho$. Since we are only interested in effects linear in the collision time we may expand $\Delta \rho$ in a first order Taylor expansion. Solving this expansion for the difference in the temperatures

$$
T_{\mathrm{R}}=T+\left(\frac{\partial \rho}{\partial T}\right)_{n}^{-1}\left[\left(\frac{\partial \rho}{\partial n}\right)_{T} \Delta n-\Delta \rho\right] .
$$

Let us now rewrite the energy-momentum tensor as calculated in the kinetic theory in terms of $T, n$ and $U$ :

$$
\begin{aligned}
& T^{k \mu \nu}(T, n)=\rho(T, n) U^{\mu} U^{\nu}-P(T, n) h^{\mu \nu} \\
& +\left[\left(\frac{\partial P}{\partial n}\right)_{\rho} \Delta n+\left(\frac{\partial P}{\partial \rho}\right)_{n} \Delta \rho-\Delta P\right] h^{\mu \nu} \\
& +T_{B}^{(1) \mu \nu}(T, n)+\frac{w}{n} U^{\mu} J_{\text {diff }}^{\nu}(T, n)+\frac{w}{n} J_{\text {diff }}^{\mu}(T, n) U^{\nu} \\
& +T_{C}^{(1) \mu \nu}(T, n) .
\end{aligned}
$$

This expression can be compared to (27) and the transport coefficients can be extracted. We express them in terms of the functions $f_{a}^{(i, j)}$,

$$
\begin{aligned}
\zeta & =f_{0}^{(1,3)}\left(\frac{\partial P}{\partial \rho}\right)_{n}^{2}+f_{0}^{(1,1)}\left(\frac{\partial P}{\partial n}\right)_{\rho}^{2} \\
& -\frac{2}{3} f_{0}^{(3,1)}\left(\frac{\partial P}{\partial \rho}\right)_{n}+\frac{1}{9} f_{0}^{(5,-1)} \\
& +2 f_{0}^{(1,2)}\left(\frac{\partial P}{\partial \rho}\right)_{n}\left(\frac{\partial P}{\partial n}\right)_{\rho}-\frac{2}{3} f_{0}^{(3,0)}\left(\frac{\partial P}{\partial n}\right)_{\rho} \\
\eta & =\frac{1}{15} f_{2}^{(5,-1)} \\
T \chi & =\frac{1}{3}\left[f_{1}^{(3,1)}-2 \frac{w}{n} f_{1}^{(3,0)}+\left(\frac{w}{n}\right)^{2} f_{1}^{(3,-1)}\right]
\end{aligned}
$$


Instead of equating the collision integral to our expansion (33) and solving for the unknown functions $\kappa_{a}(\omega, x)(a \in$ $\{0,1,2\})$ we give a qualitative correct estimate. This can be achieved by using the following model for the collision integral

$$
L\left[F^{(1)}\right] \approx-\omega \tau^{-1} F^{(1)}
$$

which corresponds to $\kappa_{a}(\omega, x)=\tau^{-1}$ for all $(a \in$ $\{0,1,2\})$. This model reflects the linear dependence on $F^{(1)}$ and generates the variation of $F^{(0)}$ in the direction of the adiabatic motion through the rate of elastic scatterings. Furthermore since neutralinos are non-relativistic at kinetic decoupling we use the following approximations

$$
f_{a}^{(i, j)} \approx f^{(i, j)} \approx i ! !(M T)^{\frac{i-3}{2}} M^{j} n \tau
$$

for odd $i$ and

$$
n \approx \frac{g}{(2 \pi)^{3 / 2}}(M T)^{3 / 2} \exp \left(\alpha-\frac{M}{T}\right) .
$$

In this case $\Delta \rho$ and $\Delta P$ depend linearly on $\Delta n: \Delta \rho \approx$ $M \Delta n, \Delta P \approx 5 / 3 T \Delta n$. At any time step $\Delta t$ the variation of the number density due to non-equilibrium processes is given by the number of collisions during $\Delta t$, i. e., $\Delta n=\dot{n} \tau$.

We find in $\mathcal{O}(\tau)$ and up to order $T / M$

$$
\eta \approx n T \tau, \quad \zeta \approx \frac{5}{3} n T \tau, \quad \chi \approx 0
$$

It is interesting to note that $\chi \approx 0$ at this order since the contribution of the transformation (67) to the energy momentum tensor cancels $T_{B}^{(1)}$.

On the first sight it might be surprising that heat conduction vanishes and bulk viscosity is nonvanishing. The mentioned cancellation between $T_{B}^{(1)}$ and $J_{\text {diff indicates }}$ that the only possible mechanism to transport heat in the neutralino fluid is convection. Since the neutralinos are very slow and very sparse heat can neither be radiated nor conducted. We decided to use the frame that is comoving with the neutralinos (Eckart's approach $\mid 13$ ), thus there is no heat conduction here. In a single fluid bulk viscosity goes along with internal degrees of freedoms or with particle production or decay. In our situation the number of neutralinos is conserved and they do not have any internal degrees of freedom which can dissipate energy. Nevertheless, the bulk viscosity is nonzero, the reason is that we are dealing with two fluids and the bulk viscosity describes the energy dissipation to the radiation fluid. There should be a corresponding term for the radiation fluid, however we can neglect this term since $\rho_{\mathrm{R}} \gg n T$ at kinetic decoupling. The authors of Ref. [11] have incorrectly assumed that $\chi \neq 0$ and $\zeta=0$ in their work. Let us note that our result $(\chi=0$ and $\zeta \neq 0)$ holds in general for any kind of WIMPy CDM.

\section{COLLISIONAL DAMPING OF ACOUSTIC PERTURBATIONS}

The viscosity coefficients and the coefficient for heat conduction enter in the decay rate of acoustic perturbations, which we will study now. Following Weinberg 15 let us start with a static homogeneous fluid with

$$
U=(1, \overrightarrow{0}), \quad \rho, P, n, T=\text { const } .
$$

This fluid should leave the adiabatic limit but stay close to thermal equilibrium. As a consequence small perturbations will occur with the space-time dependence

$$
\begin{aligned}
\delta(\rho, P, n, T, \vec{k} \cdot \vec{U})= & \left(\rho^{(1)}, P^{(1)}, n^{(1)}, T^{(1)}, \vec{k} \cdot \vec{U}^{(1)}\right) \\
& \exp (\mathrm{i} \omega \mathrm{t}) \exp (-\mathrm{i} \overrightarrow{\mathrm{k}} \cdot \overrightarrow{\mathrm{x}})
\end{aligned}
$$

Note that the perturbation of the zeroth component of $U$ should vanish in order to guarantee the normalization condition.

Inserting 80 in the conservation laws for the number density, energy density and momentum we get a system of three linear algebraic equations

$$
\mathcal{A}(\zeta, \eta)(\delta T, \delta n, \vec{k} \cdot \delta \vec{U})^{T}=\overrightarrow{0}
$$

where we used $\chi \equiv 0$ and the matrix $\mathcal{A}(\zeta, \eta)$ is given in [15]. The dispersion relation is provided by the requirement

$$
\operatorname{det} \mathcal{A}(\zeta, \eta)=0
$$

which yields to first order in the collision time $(k \equiv|\vec{k}|)$

$$
\operatorname{Re} \omega=k v_{s}, \quad \operatorname{Im} \omega=-L[\zeta, \eta] k^{2} \equiv-\Gamma[\zeta, \eta] .
$$

The square of the isentropic sound speed, $v_{s}$, is given by

$$
\begin{aligned}
v_{s}^{2} & \equiv\left(\frac{\partial P}{\partial \rho}\right)_{\sigma} \\
& =\frac{T}{\rho+P}\left(\frac{\partial P}{\partial T}\right)_{n}\left(\frac{\partial P}{\partial \rho}\right)_{n}+\frac{n}{\rho+P}\left(\frac{\partial P}{\partial n}\right)_{T} \\
& \approx \frac{5}{3} \frac{T}{M_{\tilde{\chi}}}
\end{aligned}
$$

and the characteristic length $L$ for absorption reads

$$
L[\zeta, \eta] \approx \frac{\zeta+\frac{4}{3} \eta}{2 \rho} \approx \frac{3}{2} \frac{T}{M_{\tilde{\chi}}} \tau .
$$

Note that the length scale of collisional damping is proportional to the relaxation time. The authors of [11] assume instead that the characteristic scale for acoustic absorption is given by the collision time. This is correct only if acoustic perturbations are smeared out after a single contact with the heat bath. We proved already in 
Sec. III that in the case under consideration a huge number of contacts with the heat bath is needed to establish equilibrium.

Since the parameters of the fluid are slowly varying during the cooling to $T_{\mathrm{kd}}$, the amplitude of an acoustic perturbation behaves like a WKB solution. The damping of density perturbations is given by

$$
\begin{aligned}
\delta(k) & =\exp \left(-\int_{0}^{\mathrm{t}\left(\mathrm{T}_{\mathrm{kd}}\right)} \Gamma(\mathrm{t}) \mathrm{dt}\right), \\
& =\exp \left(-\frac{3}{10} \frac{T_{\mathrm{kd}}}{M_{\tilde{\chi}}}\left(\frac{k_{\mathrm{phys}}}{H}\right)_{T=T_{\mathrm{kd}}}^{2} .\right.
\end{aligned}
$$

We integrate over the time interval $\left[0, t_{\mathrm{kd}}\right]$ during which the fluid is close to thermal equilibrium and CDM density perturbations evolve like (damped) sound waves. In principle $t_{\mathrm{kd}}$ is a function of $k$, however we find that for modes of interest we can take $t_{\mathrm{k}}$ to be independent of $k$. This follows as $\operatorname{Re}(\omega) \tau=v_{s} k_{\text {phys }} \tau<1$ is easily fulfilled for the subhorizon scales $\left(k_{\mathrm{phys}} / H\right)\left(T_{\mathrm{kd}}\right)<1 / v_{s} \sim 10^{4}$ for typical MSSM masses, including all modes of interest.

From (86) we can read off a typical wavelength for collisional damping

$$
l_{\mathrm{d}}\left(T_{\mathrm{kd}}\right)=\frac{2 \pi}{\sqrt{10}} v_{\mathrm{kd}} R_{H}\left(T_{\mathrm{kd}}\right),
$$

where $v_{\mathrm{kd}}=\sqrt{3 T_{\mathrm{kd}} / M_{\tilde{\chi}}}$ and $R_{H} \equiv 1 / H$ denotes the Hubble radius. We find $l_{\mathrm{d}} \approx 0.06(0.05) R_{H}\left(T_{\mathrm{kd}}\right)$ for $M_{\tilde{\chi}}=100(150) \mathrm{GeV}$ and $M_{\tilde{L}}=200(250) \mathrm{GeV}$.

Instead of characterizing acoustic perturbations by their wavelength or wavenumber $k_{\text {phys }} \sim a^{-1} \sim t^{\frac{1}{2}}$ it is more convenient to work with a constant in timethe rest mass $M$ of neutralinos within a sphere of radius $2 \pi / k_{\text {phys }}$ :

$$
M \equiv \frac{4}{3} \pi\left(2 \pi / k_{\text {phys }}\right)^{3} n_{\tilde{\chi}} M_{\tilde{\chi}} .
$$

Using the definition of $M$ we can write (86) as

$$
\delta(M)=\exp \left[-\left(M_{\mathrm{d}} / M\right)^{\frac{2}{3}}\right],
$$

where the mass scale of damping, $M_{\mathrm{d}}$, is given by

$$
\begin{aligned}
M_{\mathrm{d}} & =\frac{2^{4} \pi^{4}}{5}\left(\frac{3}{10}\right)^{\frac{1}{2}}\left(\frac{T_{\mathrm{kd}}}{M_{\tilde{\chi}}}\right)^{\frac{3}{2}} M_{\tilde{\chi}} n_{\tilde{\chi}}\left(T_{\mathrm{kd}}\right) R_{H}^{3}\left(T_{\mathrm{kd}}\right) \\
& \approx 2.6 \times 10^{-8} \frac{(1 \mathrm{GeV})^{3}}{\left(M_{\tilde{\chi}} T_{\mathrm{kd}}\right)^{3 / 2}} \omega_{\tilde{\chi}} M_{\odot} .
\end{aligned}
$$

Exploring the parameter space of the MSSM we find typically $M_{D} \approx 10^{-9} M_{\odot}$, cf. Fig. 田.

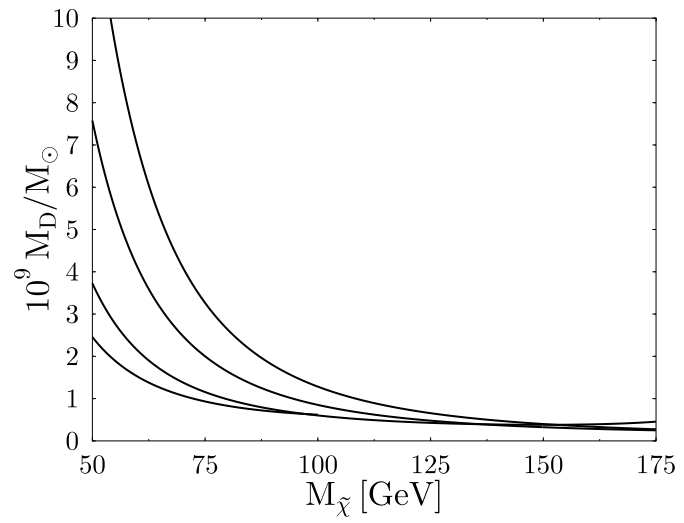

FIG. 4. The damping scale $M_{\mathrm{d}}$ in solar masses as a function of the neutralino mass $M_{\tilde{\chi}}$ for different values of the slepton Mass $M_{\tilde{L}}=150,200,300,400 \mathrm{GeV}$. The slepton mass increases from the bottom to the top.

\section{FREE STREAMING}

For temperatures $T<T_{\mathrm{ls}}$ the neutralinos are collisionless so that the viscosity coefficients vanish. Each neutralino moves along a geodesic in space-time. This geodesic motion of neutralinos provides a second damping mechanism: free streaming [23]. If the proper distance $l_{\mathrm{fs}}(t)$ which a neutralino can travel along a geodesic in time $t$ is larger then then the proper wavelength $\lambda_{\text {phys }} \equiv 2 \pi / k_{\text {phys }}$ of a perturbation at $t$ any structure will be wiped out since the neutralinos will propagate from an overdense region to an underdense region. The proper distance of free streaming for a neutralino at time $t \in\left[t_{\mathrm{ls}}, t_{\mathrm{eq}}\right]$ is given by

$$
l_{\mathrm{fs}}(a)=\frac{a_{\mathrm{ls}}}{a} v_{\mathrm{ls}} \ln \left(\frac{a}{a_{\mathrm{ls}}}\right) R_{H}(a),
$$

where $a_{\mathrm{ls}}$ denotes the expansion factor and $v_{\mathrm{ls}}=$ $\sqrt{3 T_{1 \mathrm{~s}} / M_{\tilde{\chi}}}$ the average neutralino velocity at last scattering. Exploring the parameter space of the MSSM we find at equality typically $l_{\mathrm{fs}}\left(a_{\mathrm{eq}}\right) \approx 10^{-8} R_{H}\left(a_{\mathrm{eq}}\right)$, which corresponds to $5 \times 10^{-4}$ pc today. This length scale contains a mass of $M_{\mathrm{fs}} \approx 10^{-7} M_{\odot}$, cf. Fig. 局.

Let us compare the scales of the two distinct damping mechanism at equality, the time when structures begin to grow (before equality CDM perturbations grow logarithmically). We find for $a \gg a_{\mathrm{ls}}$

$$
\frac{l_{\mathrm{fs}}}{l_{\mathrm{d}}}=\frac{\sqrt{10}}{2 \pi} \ln \left(\frac{a}{a_{\mathrm{ls}}}\right),
$$

which gives $l_{\mathrm{fs}} / l_{\mathrm{d}} \approx 6$ (or $M_{\mathrm{fs}} / M_{\mathrm{d}} \approx 220$ ) at equality. 


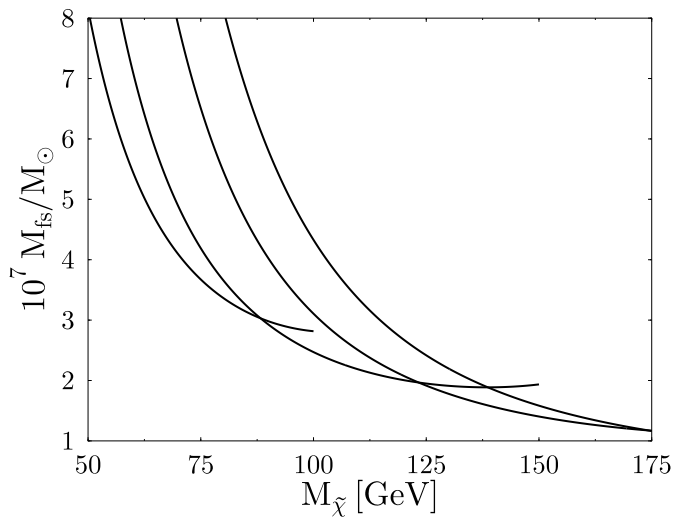

FIG. 5. The free streaming mass $M_{\mathrm{fs}}$ at matter-radiation equality in solar masses as a function of the neutralino mass $M_{\tilde{\chi}}$ for different values of the slepton mass $M_{\tilde{L}}=150,200,300,400 \mathrm{GeV}$. The slepton mass increases from the bottom to the top.

\section{CONCLUSIONS}

We have shown in this paper that kinetic decoupling of neutralino dark matter leads to collisional damping at the scale $10^{-9} M_{\odot}$. This scale could be larger for certain regions in the MSSM parameter space, e.g., when the neutralino mass and one of the slepton masses (probably the stau) are nearly degenerate. In that case our treelevel expressions become singular, and are not applicable. We have pointed out that it is important to distinguish between the collision time and the relaxation time of neutralino CDM. The corresponding temperatures differ by about an order of magnitude, which can lead to a difference of several orders of magnitude in the corresponding mass scales of damping.

The process of collisional damping has been described by imperfect fluids, and we calculated the transport coefficients from kinetic theory, by generalizing the method of Straumann [16] in order to include massive particles. We found that bulk viscosity can not be neglected, whereas heat conduction is negligible in the process of kinetic decoupling of neutralinos.

After kinetic decoupling free streaming starts to smear out remaining perturbations on scales below $10^{-7} M_{\odot}$ by the time of equality. Both scales are quite close, which shows that both mechanisms have to be considered in the calculation of the resulting power spectra for cold dark matter. We will present the corresponding transfer functions and power spectra elsewhere [24].

These damping mechanisms provide a sharp (exponential) cut-off in the power spectrum of CDM objects. Such a cut-off sets the scale for the very first objects that form in hierarchical structure formation. Although this might be impossible to observe directly, it might have implications on the substructure of galactic halos and on the structure of CDM in void regions, where some of the first CDM clouds might have a chance to survive. The cos- mological and astrophysical consequences of this cut-off will be investigated in a forthcoming publication [24].

\section{ACKNOWLEDGEMENT}

We acknowledge discussions with $\mathrm{H}$. Eberl and V.C. Spanos. D.J.S. thanks the Alexander-vonHumboldt foundation and the Austrian Academy of Sciences for financial support.

\section{APPENDIX A: CROSS SECTIONS}

This appendix contains the exact scattering amplitudes at tree level for elastic scattering and annihilation processes and simplified formulas for the related cross sections.

Let us begin with the squared transition matrix element $|\mathcal{T}|^{2}$ (summed over final and averaged over initial spins) for $\tilde{B}+\{L, \bar{L}\} \rightarrow \tilde{B}+\{L, \bar{L}\}$ expressed as a function of the usual Mandelstam variables. Our notation is as follows: $\mathcal{T}_{l}(u)$ is the scattering amplitude which describes the exchange of a left-handed slepton in the $u$ channel and so on. For the squared terms we find

$$
\begin{aligned}
& \left|\mathcal{T}_{l}(u)\right|^{2}=4\left(g b_{L}\right)^{4}\left(\frac{u-M_{\tilde{B}}^{2}-m_{L}^{2}}{u-M_{\tilde{L}}^{2}}\right)^{2}, \\
& \left|\mathcal{T}_{l}(s)\right|^{2}=4\left(g b_{L}\right)^{4}\left(\frac{s-M_{\tilde{B}}^{2}-m_{L}^{2}}{s-M_{\tilde{L}}^{2}}\right)^{2} \\
& \left|\mathcal{T}_{r}(u)\right|^{2}=4\left(g c_{L}\right)^{4}\left(\frac{u-M_{\tilde{B}}^{2}-m_{L}^{2}}{u-M_{\tilde{L}}^{2}}\right)^{2} \\
& \left|\mathcal{T}_{r}(s)\right|^{2}=4\left(g c_{L}\right)^{4}\left(\frac{s-M_{\tilde{B}}^{2}-m_{L}^{2}}{s-M_{\tilde{L}}^{2}}\right)^{2}
\end{aligned}
$$

where we assumed for simplicity that the masses of the left- and right-handed sleptons are equal.

For the different interference terms we find

$$
\begin{aligned}
& \mathcal{T}_{l}(u) \mathcal{T}_{l}^{\dagger}(s)=4\left(g b_{L}\right)^{4} \frac{2 m_{L}^{2} M_{\tilde{B}}^{2}-M_{\tilde{B}}^{2} t}{\left(u-M_{\tilde{L}}^{2}\right)\left(s-M_{\tilde{L}}^{2}\right)}, \\
& \mathcal{T}_{r}(u) \mathcal{T}_{r}^{\dagger}(s)=4\left(g c_{L}\right)^{4} \frac{2 m_{L}^{2} M_{\tilde{B}}^{2}-M_{\tilde{B}}^{2} t}{\left(u-M_{\tilde{L}}^{2}\right)\left(s-M_{\tilde{L}}^{2}\right)}, \\
& \mathcal{T}_{l}(u) \mathcal{T}_{r}^{\dagger}(u)=16 g^{4}\left(b_{L} c_{L}\right)^{2}\left(\frac{m_{L} M_{\tilde{B}}}{u-M_{\tilde{L}}^{2}}\right)^{2}, \\
& \mathcal{T}_{l}(s) \mathcal{T}_{r}^{\dagger}(s)=16 g^{4}\left(b_{L} c_{L}\right)^{2}\left(\frac{m_{L} M_{\tilde{B}}}{s-M_{\tilde{L}}^{2}}\right)^{2}, \\
& \mathcal{T}_{l}(u) \mathcal{T}_{r}^{\dagger}(s)=4 g^{4}\left(b_{L} c_{L}\right)^{2} \frac{2 m_{L}^{2} M_{\tilde{B}}^{2}-m_{L}^{2} t}{\left(u-M_{\tilde{L}}^{2}\right)\left(s-M_{\tilde{L}}^{2}\right)} .
\end{aligned}
$$


Summing up the squared and interference terms yields

$$
\begin{aligned}
|\mathcal{T}|^{2}= & 4 g^{4}\left(b_{L}^{4}+c_{L}^{4}\right) \\
& {\left[\left(\frac{s-M_{\tilde{B}}^{2}-m_{L}^{2}}{s-M_{\tilde{l}}^{2}}\right)^{2}+\left(\frac{u-M_{\tilde{B}}^{2}-m_{L}^{2}}{u-M_{\tilde{l}}^{2}}\right)^{2}\right.} \\
& \left.+\frac{\left(2 m_{L} M_{\tilde{B}}\right)^{2}-2 M_{\tilde{B}}^{2} t}{\left(s-M_{\tilde{L}}^{2}\right)\left(u-M_{\tilde{L}}^{2}\right)}\right] \\
+ & 8 g^{4}\left(b_{L} c_{L}\right)^{2} \\
& {\left[\left(\frac{2 m_{L} M_{\tilde{B}}}{s-M_{\tilde{L}}^{2}}\right)^{2}+\left(\frac{2 m_{L} M_{\tilde{B}}}{u-M_{\tilde{L}}^{2}}\right)^{2}\right.} \\
& {\left[\frac{\left(2 m_{L} M_{\tilde{B}}\right)^{2}-2 m_{L}^{2} t}{\left.\left(s-M_{\tilde{L}}^{2}\right)\left(u-M_{\tilde{L}}^{2}\right)\right]}\right.}
\end{aligned}
$$

Since $m_{L}$ denotes the mass of a standard model fermion we use $m_{L} \approx 0$. Furthermore the binos are nonrelativistic so that $s \approx M_{\tilde{B}}^{2}+2 M_{\tilde{B}} E_{L}$ to a very good approximation. Using the mentioned simplifications we find

$$
|\mathcal{T}|^{2}=256\left(b_{L}^{4}+c_{L}^{4}\right)\left(\frac{G_{\mathrm{F}} M_{W}^{2}}{M_{\tilde{L}}^{2}-M_{\tilde{B}}^{2}}\right)^{2}\left(1-\frac{t}{4 E_{L}^{2}}\right)
$$

and for the elastic cross section the simplified formula

$$
\sigma_{\mathrm{el}}\left(E_{L}\right)=\frac{24}{\pi}\left(b_{L}^{4}+c_{L}^{4}\right)\left(\frac{G_{\mathrm{F}} M_{W}^{2}}{M_{\tilde{L}}^{2}-M_{\tilde{B}}^{2}}\right)^{2} E_{L}{ }^{2} .
$$

The squared transition matrix element (summed over final and averaged over initial spins) for $\tilde{B}+\tilde{B} \rightarrow \bar{F}+F$ may be found from $|\mathcal{T}|^{2}$ for elastic scattering processes by making the following modifications: $s \rightarrow u, t \rightarrow s$ and $u \rightarrow t$. Expanding in $m_{F} / M_{\tilde{B}}$ and in the Lorentz invariant relative velocity $v$ up to second order yields

$$
\begin{aligned}
v \sigma_{\text {ann }}= & \frac{2}{\pi}\left(\frac{G_{\mathrm{F}} M_{W}^{2}}{M_{\tilde{F}}^{2}+M_{\tilde{B}}^{2}}\right)^{2}\left[\left(b_{F}^{2}+c_{F}^{2}\right)^{2} m_{F}^{2}\right. \\
& \left.+\frac{2}{3}\left(b_{F}^{4}+c_{F}^{4}\right) \frac{M_{\tilde{F}}^{4}+M_{\tilde{B}}^{4}}{\left(M_{\tilde{F}}^{2}+M_{\tilde{B}}^{2}\right)^{2}}\left(M_{\tilde{B}} v\right)^{2}\right],
\end{aligned}
$$

where $v$ is given by $(v / 2)^{2}=1-\left(2 M_{\tilde{B}}\right)^{2} / s$.

\section{APPENDIX B: ADIABATIC RELATIONS}

In this appendix we show how $\dot{T}$ and space-time gradients of $\alpha$ are related to $U_{, \lambda}^{\lambda}$ and $Q_{\mu}$.

The second law of thermodynamics gives the variation in the entropy per particle $\sigma$ as

$$
n T \mathrm{~d} \sigma=\mathrm{d} \rho-\frac{w}{n} \mathrm{~d} n .
$$

Since $\mathrm{d} \sigma$ must be a perfect differential

$$
T\left(\frac{\partial P}{\partial T}\right)_{n}=w-n\left(\frac{\partial \rho}{\partial n}\right)_{T}
$$

follows. For adiabatic motion

$$
\begin{aligned}
0 & =n T \dot{\sigma} \\
& =\left(\frac{\partial \rho}{\partial T}\right)_{n} \dot{T}-\frac{T}{n}\left(\frac{\partial P}{\partial T}\right)_{n} \dot{n},
\end{aligned}
$$

or, using the conservation law $\mathrm{d}(n U)=0$

$$
\dot{T}=-T\left(\frac{\partial P}{\partial \rho}\right)_{n} U_{, \lambda}^{\lambda} .
$$

The Gibbs-Duhem relation gives the variation of the pseudochemical potential $\alpha=\mu / T$ as

$$
\mathrm{d} \alpha=\frac{\mathrm{d} P}{n T}-\frac{w}{n T} \frac{\mathrm{d} T}{T} .
$$

For adiabatic motion and using (B2) and (B4) this yields

$$
T \dot{\alpha}=-\left(\frac{\partial P}{\partial n}\right)_{\rho} U_{, \lambda}^{\lambda} .
$$

Using (B5) and the relativistic generalization of the Euler equation we find

$$
T h^{\mu \lambda} \alpha_{, \lambda}=-\frac{w}{T n} h^{\mu \lambda} Q_{\lambda} .
$$

Thus the variation of $\alpha$ in the plane perpendicular to the adiabatic flow is generated by the projection of the heat current on this plane.

[1] P. de Bernardis et al., Nature 404, 955 (2000); S. Hanany et al., Astrophys. J. 545, L5 (2000); A. H. Jaffe et al., astro-ph/0007333.

[2] D. Tytler, et al., Phys. Scr. T85, 12 (2000).

[3] P. J. E. Peebles, Astrophys. J. 264, L1 (1982); G. R. Blumenthal et. al., Nature 311, 517 (1984).

[4] A. G. Doroshkevich, Y. B. Zel'dovich, and R. A. Sunayev, Sov. Astronom. 21, 523 (1978); M. L. Wilson and J. Silk, Astrophys. J. 243, 14 (1981); N. Kaiser, Mon. Not. R. Astr. Soc. 202, 1169 (1983); S. D. M. White, C. Frenk, and M. Davis, Astrophys. J. 274, L1 (1983).

[5] S. S. McGaugh, Astrophys. J. 541, L33 (2000).

[6] L. M. Griffiths, A. Melchiorri, and J. Silk, astro-ph/0101413. 
[7] The scale invariance of hierarchical structure formation obviously has to be broken at some small scale, otherwise the contribution of density fluctuations to the local energy density would be ultra-violet singular.

[8] See, e.g., G. Jungmann, M. Kaminokowski and K. Griest, Phys. Rep. 267, 195 (1996).

[9] C. Schmid, D. J. Schwarz and P. Widerin, Phys. Rev. D 59, 043517 (1999).

[10] D. J. Schwarz and S. Hofmann, Nucl. Phys. Proc. Suppl. 87, 93 (2000), astro-ph/9912343.

[11] C. Boehm, P. Fayet and R. Schaeffer, astro-ph/0012504.

[12] X. Chen, M. Kamionkowski, and X. Zhang, astro-ph/0103452.

[13] C. Eckart, Phys. Rev. 58, 919 (1940).

[14] L. D. Landau and E. M. Lifschitz, Hydrodynamik (Akademie Verlag, Berlin, 1991).

[15] S. Weinberg, Astrophys. J. 168, 175 (1971).

[16] N. Straumann, Helv. Phys. Acta 49, 269 (1976).

[17] R. Barate et al., ALEPH Collaboration, Phys. Lett. B 499, 67 (2001).

[18] J. Ellis, T. Falk, K. A. Olive and M. Schmitt, Phys. Lett. B 413, 355 (1997) hep-ph/9705444; J. Ellis et al., Phys. Rev. D 58, 095002 (1998) hep-ph/9801445; J. Ellis, T. Falk and K. A. Olive, Phys. Lett. B 444, 367 (1998) hep-ph/9810360; J. Ellis, T. Falk, K. A. Olive and M. Srednicki, Astropart. Phys. 13, 181 (2000) hepph/9905481]; J. Ellis, T. Falk, G. Ganis and K. A. Olive, Phys. Rev. D 62, 075010 (2000) hep-ph/0004169; J. Ellis et al., hep-ph/0102098.

[19] K. Griest, M. Kamionkowski, and M. S. Turner, Phys. Rev. D 41, 3565 (1990).

[20] K. Griest and D. Seckel, Phys. Rev. D 43, 3191 (1991); P. Gondolo and G. Gelmini, Nucl. Phys. B 360, 145 (1991); M. Drees and M.M. Nojiri, Phys. Rev. D 47, 376 (1993); H. Baer and M. Brhlik, Phys. Rev. D 53, 597 (1996); V. Barger and C. Kao, Phys. Rev. D 57, 3131 (1998); A. B. Lahanas, D. V. Nanopoulos and V. C. Spanos, Phys. Lett. B 464, 213 (1999) hepph/9906394; A. B. Lahanas, D. V. Nanopoulos and V. C. Spanos, Phys. Rev. D 62, 023515 (2000) hep$\mathrm{ph} / 9909497$.

[21] K. Griest, Phys. Rev. D 38, 2357 (1988); Erratum 39, 3802 (1989).

[22] H. Sato, T. Mabuda and H. Takeda, Progr. Theor. Phys. Suppl. 49, 11 (1971), Appendix G; I. Masaki, Publ. Astron. Soc. Japan 23, 425 (1971).

[23] J. R. Bond and A. S. Szalay, Astrophys. J. 274, 443 (1983).

[24] S. Hofmann and D. J. Schwarz, in preparation. 